\title{
A Sensitive Cardiac Troponin T Assay in Stable Coronary Artery Disease
}

Torbjørn Omland, M.D., Ph.D., M.P.H., James A. de Lemos, M.D., Marc S. Sabatine, M.D., M.P.H., Costas A. Christophi, Ph.D., Madeline Murguia Rice, Ph.D., Kathleen A. Jablonski, Ph.D., Solve Tjora, M.D., Michael J. Domanski, M.D., Bernard J. Gersh, M.B., Ch.B., D.Phil., Jean L. Rouleau, M.D., Marc A. Pfeffer, M.D., Ph.D., Eugene Braunwald, M.D., and the Prevention of Events with Angiotensin Converting Enzyme Inhibition (PEACE) Trial Investigators

Division of Medicine (T.O.) and the Center of Laboratory Medicine (S.T.), Akershus University Hospital, Lørenskog, Norway; the Faculty Division Akershus University Hospital and the Center for Heart Failure Research, University of Oslo, Oslo (T.O.); the Department of Medicine, University of Texas Southwestern Medical Center, Dallas (J.A.L.); the Department of Medicine, Harvard Medical School, and the Cardiovascular Division, Brigham and Women's Hospital both in Boston (M.S.S., M.A.P., E.B.); the Biostatistics Center, George Washington University, Rockville, MD (C.A.C., M.M.R., K.A.J.); National Heart, Lung, and Blood Institute, Bethesda, MD (M.J.D.); the Division of Cardiovascular Diseases, Department of Medicine, Mayo Clinic, Rochester, MN (B.J.G.); and Montreal Heart Institute, and Faculty of Medicine, University of Montreal — both in Montreal (J.L.R.).

\section{Abstract \\ BACKGROUND—In most patients with stable coronary artery disease, plasma cardiac troponin $\mathrm{T}$ levels are below the limit of detection for the conventional assay. The distribution and determinants of very low circulating troponin T levels, as well as their association with cardiovascular events, in such patients are unknown.}

METHODS-We used a new, high-sensitivity assay to determine the concentration of cardiac troponin $\mathrm{T}$ in plasma samples from 3679 patients with stable coronary artery disease and preserved left ventricular function. Results of the assay were analyzed in relation to the incidence of cardiovascular events during a median follow-up period of 5.2 years.

RESULTS-With the highly sensitive assay, concentrations of cardiac troponin T were at or above the limit of detection ( $0.001 \mu \mathrm{g}$ per liter) in 3593 patients (97.7\%) and at or above the 99th percentile for apparently healthy subjects $(0.0133 \mu \mathrm{g}$ per liter) in 407 patients $(11.1 \%)$. After adjustment for other independent prognostic indicators, there was a strong and graded increase in the cumulative incidence of cardiovascular death (adjusted hazard ratio per unit increase in the natural logarithm of the troponin T level, 2.09; 95\% confidence interval [CI], 1.60 to 2.74; $\mathrm{P}<0.001$ ) and of heart failure (adjusted hazard ratio, 2.20; 95\% CI, 1.66 to $2.90 ; \mathrm{P}<0.001$ ) in this study group. Increased risk associated with higher levels of troponin $\mathrm{T}$ was evident well below the limit of detection of conventional cardiac troponin $\mathrm{T}$ assays and below the 99th percentile of values in a healthy population. There was no association between troponin $\mathrm{T}$ levels as measured with the highly sensitive assay and the incidence of myocardial infarction (adjusted hazard ratio, $1.16 ; 95 \% \mathrm{CI}, 0.97$ to $1.40 ; \mathrm{P}=0.11$ ).

Copyright (@) 2009 Massachusetts Medical Society. All rights reserved.

Address reprint requests to Dr. Omland at the Division of Medicine, Akershus University Hospital, NO-1478 Lørenskog, Norway, or at torbjorn.omland@medisin.uio.no.. 
CONCLUSIONS—After adjustment for other independent prognostic indicators, cardiac troponin $\mathrm{T}$ concentrations as measured with a highly sensitive assay were significantly associated with the incidence of cardiovascular death and heart failure but not with myocardial infarction in patients with stable coronary artery disease.

Cardiac troponins tand are components of the contractile apparatus of cardiomyocytes and are the preferred biochemical markers of myocardial necrosis in patients with suspected acute coronary syndromes. ${ }^{1}$ Among such patients, a strong association between elevated troponin levels and recurrent coronary ischemic events has been firmly established. ${ }^{2-4}$

It has been shown that even very small elevations in troponins are associated with an increased risk of an adverse outcome in patients with acute coronary syndromes..$^{5}$ Moreover, among men clinically free of cardiovascular disease, as well as in patients with recent acute coronary syndromes, levels of cardiac troponin greater than $0.01 \mu \mathrm{g}$ per liter have been associated with increased mortality. ${ }^{6,7}$ Thus, it seems plausible that cardiac troponin levels below the conventional limits of detection may further discriminate between subjects at high risk and those at low risk for future cardiovascular events.

A highly sensitive assay for cardiac troponin $\mathrm{T}$ has recently been developed, permitting measurement of concentrations that are lower by a factor of 10 than those measurable with conventional assays. We hypothesized that with the highly sensitive assay, cardiac troponin $\mathrm{T}$ would be detectable in patients who had stable coronary artery disease without heart failure or left ventricular systolic dysfunction and that these levels would be associated with the risk of future cardiovascular events.

\section{METHODS}

\section{PATIENTS}

This substudy involved patients who had been included in the Prevention of Events with Angiotensin Converting Enzyme Inhibition (PEACE) Trial (ClinicalTrials.gov number, NCT00000558). The design, entry criteria, and main results of this trial have been described previously. ${ }^{8}$ In brief, from November 1996 through June 2000, a total of 8290 patients with stable coronary artery disease and preserved left ventricular systolic function were randomly assigned to receive either the angiotensin-converting-enzyme inhibitor trandolapril or placebo. All the participants were free of heart failure at baseline, and none had been hospitalized for unstable angina within the 3 months preceding trial entry. (For details about the inclusion and exclusion criteria, see Table 1 in the Supplementary Appendix, available with the full text of this article at NEJM.org.) Patients were followed every 6 months until December 2003, for a median of 5.2 years. The PEACE trial was sponsored by the National Heart, Lung, and Blood Institute (NHLBI) with support from Knoll Pharmaceuticals and Abbott Laboratories. It was conducted after approval by ethics committees from all participating sites, and patients provided written informed consent.

All participants from the United States and Canada were eligible for biospecimen collection, and approximately half the participants agreed to provide a baseline blood sample. All patients who had baseline EDTA-treated plasma samples for measurement of troponin $\mathrm{T}$ were included in this substudy.

\section{BIOMARKER SUBSTUDY}

This biomarker study was designed by the first author and supported by Roche Diagnostics. Clinical data were collected by the clinical sites; biomarkers were assayed by two of the authors in 2007. The data were held and analyzed by the clinical and statistical coordinating center at George Washington University, Rockville, Maryland. The first author wrote the 
first draft of the manuscript, which was revised by all the authors. The NHLBI project officers (but not Knoll Pharmaceuticals, Abbott Laboratories, or Roche Diagnostics) played a role in the study design; collection, analysis, and interpretation of data; the writing of the report; and the decision to submit the manuscript for publication. The first author and the coordinating center take responsibility for the accuracy and completeness of the data presented.

Before randomization, EDTA-treated plasma samples were obtained from trial participants and centrifuged at room temperature. The plasma component was aspirated and frozen at $-20^{\circ} \mathrm{C}$ at individual centers. Within 3 months after collection, plasma samples were shipped on dry ice to the central laboratory for storage at or below $-70^{\circ} \mathrm{C}$, pending analysis.

\section{BIOCHEMICAL ANALYSIS}

Levels of cardiac troponin $\mathrm{T}$ were determined by means of an autoanalyzer (cobas e 411, Roche Diagnostics). The lower limit of detection of the pre-commercial highly sensitive assay was $0.001 \mu \mathrm{g}$ per liter. ${ }^{9}$ The value at the 99th percentile in a sample of 1338 apparently healthy blood donors was $0.0133 \mu \mathrm{g}$ per liter (Roche Diagnostics, data on file). The detection limit of the older Roche troponin $\mathrm{T}$ assay (the fourth-generation Elecsys troponin $\mathrm{T}$ assay) is $0.01 \mu \mathrm{g}$ per liter; according to the manufacturer, this corresponds to a value of $0.03 \mu \mathrm{g}$ per liter with the new assay. (Details regarding the characteristics of the newer assay are provided in the Supplementary Appendix.)

$\mathrm{N}$-terminal pro-brain natriuretic peptide (NT-proBNP) concentrations were determined with an electrochemiluminescence immunoassay on a Modular platform (Roche Diagnostics). 10 C-reactive protein (CRP) measurements were performed with the highly sensitive CRPLatex (II) immunoturbidimetric assay (Denka Seiken) on a Hitachi 911 immunoanalyzer (Roche Diagnostics).11 The glomerular filtration rate was estimated with the use of the fourvariable Modification of Diet in Renal Disease (MDRD) equation.12 All biochemical testing was performed by study personnel who were unaware of the clinical outcomes and treatment assignments in the main study.

\section{OUTCOMES}

The outcomes examined in this analysis included cardiovascular death, fatal and nonfatal heart failure, and fatal and nonfatal acute myocardial infarction. All events were confirmed by a review of medical records. Cardiovascular death and fatal and nonfatal myocardial infarction were prespecified end points of the PEACE trial that underwent blinded review by an outcomes committee. Heart failure was classified by centrally trained local staff and confirmed by staff at the coordinating center through a review of hospital records. For an event to be classified as nonfatal heart failure, hospitalization with a primary cause of heart failure was required. All clinical events were classified before biomarkers were measured.

\section{STATISTICAL ANALYSIS}

Baseline characteristics are reported as percentages for categorical variables and means $\pm \mathrm{SD}$ for continuous variables. We used analysis-of-variance and chi-square tests to test for differences in continuous and categorical baseline characteristics between sex-specific quartiles of cardiac troponin T levels. The overall F-test is reported for the analysis-ofvariance models. Pearson's correlation was used to describe the association between the natural logarithm of the troponin $\mathrm{T}$ level and quantitative risk factors (after logarithmic transformation, when appropriate).

Cox proportional-hazards models were used to examine the association between troponin $\mathrm{T}$ levels as determined by the highly sensitive assay and outcome events; data were censored 
at the time of the last visit. In these models, the troponin $\mathrm{T}$ level was examined both as a continuous variable (after logarithmic transformation) and as a categorical variable by sexspecific quartiles. Cox models sequentially added treatment group, age, sex, smoking status, body-mass index, ejection fraction, glomerular filtration rate, total cholesterol level, presence or absence of a history of various cardiovascular events or conditions (hypertension, myocardial infarction, diabetes mellitus, stroke, percutaneous coronary intervention, or coronary-artery bypass grafting), and medications (beta-blockers, lipidlowering drugs, diuretics, and aspirin or antiplatelet drugs). The collinearity index was used to check for linear combinations among covariates. ${ }^{13}$

The final model was adjusted for treatment assignment and for baseline variables that are significantly associated with cardiovascular death (age, sex, and current smoking status), as well as for CRP values and NT-proBNP levels. We tested for an interaction between treatment assignment and the effect of troponin $\mathrm{T}$ level on the outcome by adding interaction terms in the models. Nelson-Aalen curves were generated to show the cumulative incidence of cardiovascular death for each quartile of troponin $\mathrm{T}$.

Estimates of the $\mathrm{C}$ statistic for the Cox regression models were calculated with the method described by Pencina and D'Agostino. ${ }^{14}$ Covariate-adjusted receiver-operating-characteristic plots were also generated for models both with and without high-sensitivity cardiac troponin $\mathrm{T}$. The increased discriminative value of the troponin $\mathrm{T}$ level was examined by using the integrated discrimination improvement (IDI) measure ${ }^{15}$ to estimate the change in the predictive survival probabilities for cardiovascular death. Discrimination slopes were compared between models with and without the troponin $\mathrm{T}$ data. We assessed model calibration by comparing predicted probabilities with observed probabilities.16 Residual analysis was used to assess model fit.

A P value of less than 0.05 was considered to indicate statistical significance, and all tests were two-sided. No adjustment for multiple comparisons was performed. The SAS analysis system, version 9.1, was used for all analyses (SAS Institute).

\section{RESULTS}

\section{BASELINE CHARACTERISTICS OF THE PATIENTS}

Baseline troponin T measurements were available from 3679 patients at 187 clinical sites. Characteristics of the patients included in the main PEACE study and in the biomarker substudy are summarized in Table 2 in the Supplementary Appendix. There were no clinically relevant differences between patients included in the substudy and those not included in it. The mean $( \pm \mathrm{SD})$ age of patients in the substudy was $63.6 \pm 8.2$ years, $18.9 \%$ were women, and $91.6 \%$ were white (Table 1). All patients had stable coronary artery disease, and $56.3 \%$ had a history of myocardial infarction, $45.6 \%$ of percutaneous coronary intervention, and $35.8 \%$ of coronary-artery bypass grafting.

\section{DISTRIBUTION AND DETERMINANTS OF VERY LOW LEVELS OF TROPONIN T}

Concentrations of troponin $\mathrm{T}$ as measured with the highly sensitive assay were at or above the limit of detection $(0.001 \mu \mathrm{g}$ per liter) in 3593 patients $(97.7 \%)$ and equal to or greater 99th percentile value for apparently healthy subjects $(0.0133 \mu \mathrm{g}$ per liter $)$ in 407 patients $(11.1 \%)$. The distribution of troponin $\mathrm{T}$ concentrations in patients with stable coronary artery disease and in apparently healthy blood donors is shown in Figure 1. Troponin $\mathrm{T}$ levels were higher in men than in women (median, 0.0063 vs. $0.0046 \mu$ g per liter; $\mathrm{P}<0.001$ ). Characteristics of the patients according to sex-specific quartiles of troponin $\mathrm{T}$ values are shown in Table 1. Levels of troponin $\mathrm{T}$ were positively associated with several conventional risk factors. 


\section{TROPONIN T AND CARDIOVASCULAR EVENTS}

Cardiovascular Deaths-During follow-up, there were 125 cardiovascular deaths in the biomarker substudy cohort. In the univariate model, the cumulative incidence of cardiovascular deaths was significantly associated with the cardiac troponin T level (hazard ratio per unit increase in the natural logarithm of the value for troponin $\mathrm{T}, 2.78 ; 95 \%$ confidence interval $[\mathrm{CI}], 2.24$ to $3.45 ; \mathrm{P}<0.001$ ) (Table 2). After adjustment for age, sex, smoking status, and high-sensitivity CRP level by using the multivariate model 1 , the association remained strong (hazard ratio, 2.39; 95\% CI, 1.85 to 3.09; $\mathrm{P}<0.001$ ). The addition of NT-proBNP as a covariate in the multivariate model modestly attenuated the association, but the relation still remained highly significant (hazard ratio, 2.09; 95\% CI, 1.60 to $2.74 ; \mathrm{P}<0.001)$.

The risk of cardiovascular death associated with increasing quartiles of troponin $\mathrm{T}$ was also strong and graded $(\mathrm{P}<0.001$ for trend) (Fig. 2). This trend remained significant $(\mathrm{P}<0.001)$ after multivariate adjustment (Table 3 in the Supplementary Appendix). Moreover, when the first quartile was used as the reference category, the adjusted association with risk was significant for both the third and fourth quartiles of troponin T (Fig. 1 in the Supplementary Appendix). When the first decile was used as the reference, the hazard ratio point estimates were only slightly increased from the second to the fifth decile, with an apparent threshold effect corresponding to the sixth decile (Fig. 2 in the Supplementary Appendix). Since only 407 patients had levels above the 99th percentile for apparently healthy subjects $(0.0133 \mu \mathrm{g}$ per liter - i.e., within the ninth decile), the troponin T levels determined by the highly sensitive assay appear to provide independent prognostic information below this limit.

Of the 3679 participants, 3630 had troponin T levels that would be expected to be undetectable by the conventional assay (i.e., $<0.01 \mu \mathrm{g}$ per liter, which corresponds to $<0.03$ $\mu \mathrm{g}$ per liter with the new assay). In this subgroup, 117 cardiovascular deaths occurred. The risk estimate for these 3630 patients was similar to that for the entire cohort (hazard ratio, 2.14; $95 \% \mathrm{CI}, 1.52$ to $3.04 ; \mathrm{P}<0.001$ ), suggesting that the newer troponin $\mathrm{T}$ assay provides powerful prognostic information in patients who would have undetectable levels with the conventional assay.

Heart Failure-During follow-up there were 104 first hospitalizations for fatal or nonfatal heart failure. In unadjusted analyses, the incidence of heart failure increased with increasing levels of troponin $\mathrm{T}$ (Table 2 ). After adjustment, the association remained highly significant (hazard ratio, 2.20; 95\% CI, 1.66 to $2.90 ; \mathrm{P}<0.001$ ). The risk of heart failure associated with increasing quartiles of troponin $\mathrm{T}$ levels was also strong and graded in both univariate and multivariate analyses ( $\mathrm{P}<0.001$ for trend) (Table 3 in the Supplementary Appendix).

Cardiovascular Deaths Not Due to Heart Failure-There were 112 cardiovascular deaths classified as not being due to heart failure during follow-up. In an unadjusted analysis, the cumulative incidence of this end point was significantly associated with troponin $\mathrm{T}$ (Table 2). After adjustment, the association was modestly attenuated but remained highly significant (hazard ratio, $1.95 ; 95 \% \mathrm{CI}, 1.46$ to $2.61 ; \mathrm{P}<0.001$ ). The risk of this outcome in association with increasing quartiles of troponin $\mathrm{T}$ levels was also strong and graded above the second quartile $(\mathrm{P}<0.001$ for trend) (Table 3 in the Supplementary Appendix).

Myocardial Infarction-During follow-up, there were 233 fatal or nonfatal acute myocardial infarctions. In an unadjusted analysis, there was a weak but significant increase in the cumulative incidence of myocardial infarction with increasing troponin $\mathrm{T}$ levels (Table 2). After adjustment, this association was no longer significant (hazard ratio, 1.16; 
$95 \% \mathrm{CI}, 0.97$ to $1.40 ; \mathrm{P}=0.11$ ). Likewise, an unadjusted analysis showed a weak association between the risk of myocardial infarction and increasing quartiles of troponin $\mathrm{T}$ that was of borderline significance; after adjustment for potentially confounding variables, this association was no longer significant $(\mathrm{P}=0.54)$ (Table 3 in the Supplementary Appendix).

\section{DISCRIMINATION, RECLASSIFICATION, AND CALIBRATION}

For the end point of cardiovascular death, the $\mathrm{C}$ statistic of the sequential model that included high-sensitivity CRP was 0.70 . Adding troponin $\mathrm{T}$ to this model increased the $\mathrm{C}$ statistic to 0.75 ( $\mathrm{P}<0.001)$ (Fig. 3A). The $\mathrm{C}$ statistic of the sequential model that included high-sensitivity CRP and NT-proBNP was 0.74 . Adding troponin $\mathrm{T}$ to this model modestly but significantly increased the $\mathrm{C}$ statistic, to $0.76(\mathrm{P}=0.007)$ (Fig. 3B).

The IDI for the end point of cardiovascular death was calculated. Inclusion of troponin $\mathrm{T}$ in the final sequential model that also included high-sensitivity CRP resulted in a significant improvement in performance of the model (IDI, 0.018; $\mathrm{P}<0.001$ ). This was also true for the model that included both high-sensitivity CRP and NT-proBNP (IDI, 0.014; $\mathrm{P}<0.001$ ). Calibration of the models with and without troponin $\mathrm{T}$ showed no evidence of lack of fit.

\section{DISCUSSION}

This study shows that very low circulating levels of cardiac troponin $\mathrm{T}$ are detectable in the great majority of patients who have stable coronary artery disease with preserved left ventricular function, that multiple conventional risk factors are associated with higher troponin $\mathrm{T}$ concentrations in this population, and that very low circulating levels of troponin $\mathrm{T}$ have a graded relationship with the incidence of cardiovascular death and heart failure in such patients. Conversely, the association between troponin $\mathrm{T}$ concentrations and incident myocardial infarction was not significant after adjustment for potential confounding factors. This finding stands in stark contrast to observations in patients with acute coronary syndromes, in whom troponins are considered biomarkers of acute cardiovascular injury due to plaque rupture and intracoronary thrombosis ${ }^{17}$ and more accurately predict recurrent myocardial infarction than they do death or heart-failure events. ${ }^{18}$

With the use of conventional assays, the prevalence of detectable concentrations of cardiac troponin $\mathrm{T}$ in the general population is approximately $0.7 \% .{ }^{19}$ Detectable troponin $\mathrm{T}$ levels in such a population are typically associated with established cardiovascular disease, such as left ventricular hypertrophy or dysfunction, or with high-risk conditions such as kidney disease or diabetes. The mechanisms responsible for the release of very low levels of cardiac troponin $\mathrm{T}$ in patients with stable coronary artery disease could include transient, clinically silent ischemic episodes and small-vessel occlusions; inflammatory processes; cardiomyocyte apoptosis ${ }^{20}$; reduced renal clearance; and increased myocardial strain due to pressure or volume overload. However, the remarkably high prevalence of detectable troponin in this low-risk cohort raises the possibility that some troponin may circulate under normal circumstances in human plasma. Data from population studies will be needed to confirm this hypothesis, and the source of the release of cardiac troponin $\mathrm{T}$ will need to be studied in experimental models.

In a previous study, the prognostic value of the highly sensitive cardiac troponin $\mathrm{T}$ assay was investigated in patients with chronic symptomatic heart failure and systolic dysfunction. ${ }^{9}$ A strong and graded association with mortality and the risk of heart failure was observed, even after adjustment for NT-proBNP. The current study extends these findings to a lowerrisk population with stable coronary artery disease and no evidence of heart failure or left ventricular systolic dysfunction. 
The observations that troponin $\mathrm{T}$ was detectable in almost all our patients and that it provided strong prognostic information independently of conventional risk factors and other contemporary biomarkers, such as NT-proBNP and high-sensitivity CRP, suggest that assessment of low-level chronic myocardial injury may represent a new means by which clinicians can stratify risk among patients with stable coronary artery disease and preserved left ventricular function. Unresolved questions that need to be addressed include whether serial testing would enhance the prognostic value of the assay and whether minor changes in very low levels are stronger predictors of events than are absolute values. Moreover, before routine testing is considered, the therapeutic implications will need to be fully explored. Certain negative implications merit consideration as well. As more sensitive assays for troponins are introduced clinically, the specificity of low-level troponin elevation for acute myocardial injury in patients with acute chest pain syndromes may be reduced.

Limitations of our study include the selection of patients participating in a clinical trial (who may not be fully representative of patients in the general population), the use of a noncommercially available prototype assay that may not be perfectly calibrated with existing conventional assays, and the fact that heart-failure events were not a component of the prespecified primary outcome in the original trial design. In addition, we cannot rule out the presence of asymptomatic cardiovascular disease in the group of apparently healthy blood donors whose troponin $\mathrm{T}$ values were used for comparison, although anyone who was receiving medication for cardiovascular disease was excluded from this group. Accordingly, we cannot rule out the possibility of a lower 99th percentile value with the use of a more rigorous screening procedure. Moreover, the PEACE study population was predominantly white, and more than $80 \%$ of the patients were men. Therefore, extrapolation of our results to other demographic groups should be done with caution.

In conclusion, very low levels of troponin $\mathrm{T}$ were detectable in the great majority of patients with stable coronary artery disease. Levels well below the limit of detection of previous assays and below the 99th percentile in apparently healthy blood donors were strongly associated with the incidence of cardiovascular death and heart failure. In contrast, troponin $\mathrm{T}$ levels were not independently associated with the incidence of myocardial infarction.

\section{Supplementary Material}

Refer to Web version on PubMed Central for supplementary material.

\section{Acknowledgments}

Dr. Omland reports receiving lecture fees and grant support from Abbott Laboratories and Roche Diagnostics; Dr. de Lemos, grant support and consulting fees from Biosite/Inverness and Roche Diagnostics, lecture fees from Roche Diagnostics, and consulting fees from Tethys Bioscience and Johnson \& Johnson; Dr. Sabatine, grant support from Roche Diagnostics, Singulex, diaDexus, and Ortho Clinical Diagnostics and consulting fees from Singulex; Drs. Christophi, Rice, and Jablonski, grant support from Knoll Pharmaceuticals and Abbott Laboratories; Dr. Gersh, receiving consulting fees from Amorcyte, AstraZeneca, Bristol-Myers Squibb, Boston Scientific, and Abbott Laboratories, and having equity ownership in CV Therapeutics; Dr. Rouleau, receiving consulting fees from Novartis and NicOx, lecture fees from Pfizer and Novartis, and grant support from Scios; and Dr. Pfeffer, receiving grant support from Abbott, Amgen, Atherogenics, Novartis, and Sanofi-Aventis, lecture fees from Bristol-Myers Squibb and Pfizer, and consulting fees from AstraZeneca, BMS, Genzyme, Novartis, and Pfizer and being listed as a coinventor on patents regarding the use of inhibition of the renin-angiotensin system that are licensed to Abbott and Novartis. No other potential conflict of interest relevant to this article was reported.

We thank the PEACE investigators, research coordinators, and committee members and Ms. Nina Samuelsen. 


\section{References}

1. Gupta S, de Lemos JA. Use and misuse of cardiac troponins in clinical practice. Prog Cardiovasc Dis 2007;50:151-65. [PubMed: 17765476]

2. Hamm CW, Ravkilde J, Gerhardt W, et al. The prognostic value of serum troponin $\mathrm{T}$ in unstable angina. N Engl J Med 1992;327:146-50. [PubMed: 1290492]

3. Lindahl B, Toss H, Siegbahn A, Venge P, Wallentin L. Markers of myocardial damage and inflammation in relation to long-term mortality in unstable coronary artery disease. N Engl J Med 2000;343:1139-47. [PubMed: 11036119]

4. Antman EM, Tanasijevic MJ, Thompson B, et al. Cardiac-specific troponin I levels to predict the risk of mortality in patients with acute coronary syndromes. N Engl J Med 1996;335:1342-9. [PubMed: 8857017]

5. James S, Armstrong P, Califf R, et al. Troponin T levels and risk of 30-day outcomes in patients with the acute coronary syndrome: prospective verification in the GUSTO-IV trial. Am J Med 2003;115:178-84. [PubMed: 12935823]

6. Zethelius B, Johnston N, Venge P. Troponin I as a predictor of coronary heart disease and mortality in 70-year-old men: a community-based cohort study. Circulation 2006;113:1071-8. [PubMed: 16490824]

7. Eggers KM, Lagerqvist B, Venge P, Wallentin L, Lindahl B. Persistent cardiac troponin I elevation in stabilized patients after an episode of acute coronary syndrome predicts long-term mortality. Circulation 2007;116:1907-14. [PubMed: 17909103]

8. The PEACE Trial Investigators. Angiotensin-converting-enzyme inhibition in stable coronary artery disease. N Engl J Med 2004;351:2058-68. [PubMed: 15531767]

9. Latini R, Masson S, Anand IS, et al. Prognostic value of very low plasma concentrations of troponin $\mathrm{T}$ in patients with stable chronic heart failure. Circulation 2007;116:1242-9. [PubMed: 17698733]

10. Omland T, Sabatine MS, Jablonski KA, et al. Prognostic value of B-type natriuretic peptides in patients with stable coronary artery disease: the PEACE Trial. J Am Coll Cardiol 2007;50:205-14. [PubMed: 17631211]

11. Roberts WL, Moulton L, Law TC, et al. Evaluation of nine automated high-sensitivity C-reactive protein methods: implications for clinical and epidemiological applications. Clin Chem 2001;47:418-25. [PubMed: 11238291] [Erratum, Clin Chem 2001;47:980.]

12. Levey AS, Bosch JP, Lewis JB, Greene T, Rogers N, Roth D. A more accurate method to estimate glomerular filtration rate from serum creatinine: a new prediction equation. Ann Intern Med 1999;130:461-70. [PubMed: 10075613]

13. Belsley, DA.; Kuh, E.; Welsch, RE. Regression diagnostics: identifying influential data and sources of collinearity. John Wiley; New York: 1980.

14. Pencina MJ, D'Agostino RB. Overall C as a measure of discrimination in survival analysis: model specific population value and confidence interval estimation. Stat Med 2004;23:2109-23. [PubMed: 15211606]

15. Pencina MJ, D'Agostino RB, D'Agostino RB Jr, Vasan RS. Evaluating the added predictive ability of a new marker: from area under the ROC curve to reclassification and beyond. Stat Med 2008;27:157-72. [PubMed: 17569110]

16. Gronnesby JK, Borgan O. A for checking regression models in survival analysis based on the risk score. Lifetime Data Anal 1996;2:315-28. [PubMed: 9384628]

17. Wong GC, Morrow DA, Murphy S, et al. Elevations in troponin T and I are associated with abnormal tissue level perfusion: a TACTICS-TIMI 18 substudy: Treat Angina with Aggrastat and Determine Cost of Therapy with an Invasive or Conservative Strategy-Thrombolysis in Myocardial Infarction. Circulation 2002;106:202-7. [PubMed: 12105159]

18. Lindahl B, Diderholm E, Lagerqvist B, Venge P, Wallentin L. Mechanisms behind the prognostic value of troponin $\mathrm{T}$ in unstable coronary artery disease: a FRISC II substudy. J Am Coll Cardiol 2001;38:979-86. [PubMed: 11583868]

19. Wallace TW, Abdullah SM, Drazner MH, et al. Prevalence and determinants of troponin T elevation in the general population. Circulation 2006;113:1958-65. [PubMed: 16618821] 
20. Olivetti G, Abbi R, Quaini F, et al. Apoptosis in the failing human heart. N Engl J Med 1997;336:1131-41. [PubMed: 9099657] 


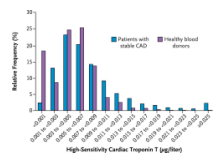

Figure 1.

Distribution of High-Sensitivity Cardiac Troponin T in Patients with Stable Coronary Artery Disease (CAD) and in Apparently Healthy Blood Donors. 


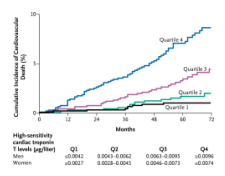

Figure 2.

Incidence of Cardiovascular Death According to Quartile of High-Sensitivity Cardiac Troponin T Level. 


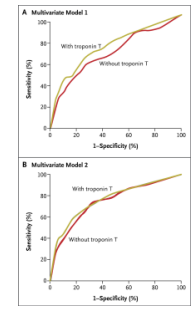

Figure 3. Covariate-Adjusted Receiver-Operating-Characteristic Plots for the Use of HighSensitivity Cardiac Troponin T to Predict the Risk of Cardiovascular Death

Panel A shows the curves for the model adjusted for treatment assignment, age, sex, smoking status, and high-sensitivity C-reactive protein level. Panel B shows the curves for the model that included these with the addition of the $\mathrm{N}$-terminal pro-brain natriuretic peptide level. 


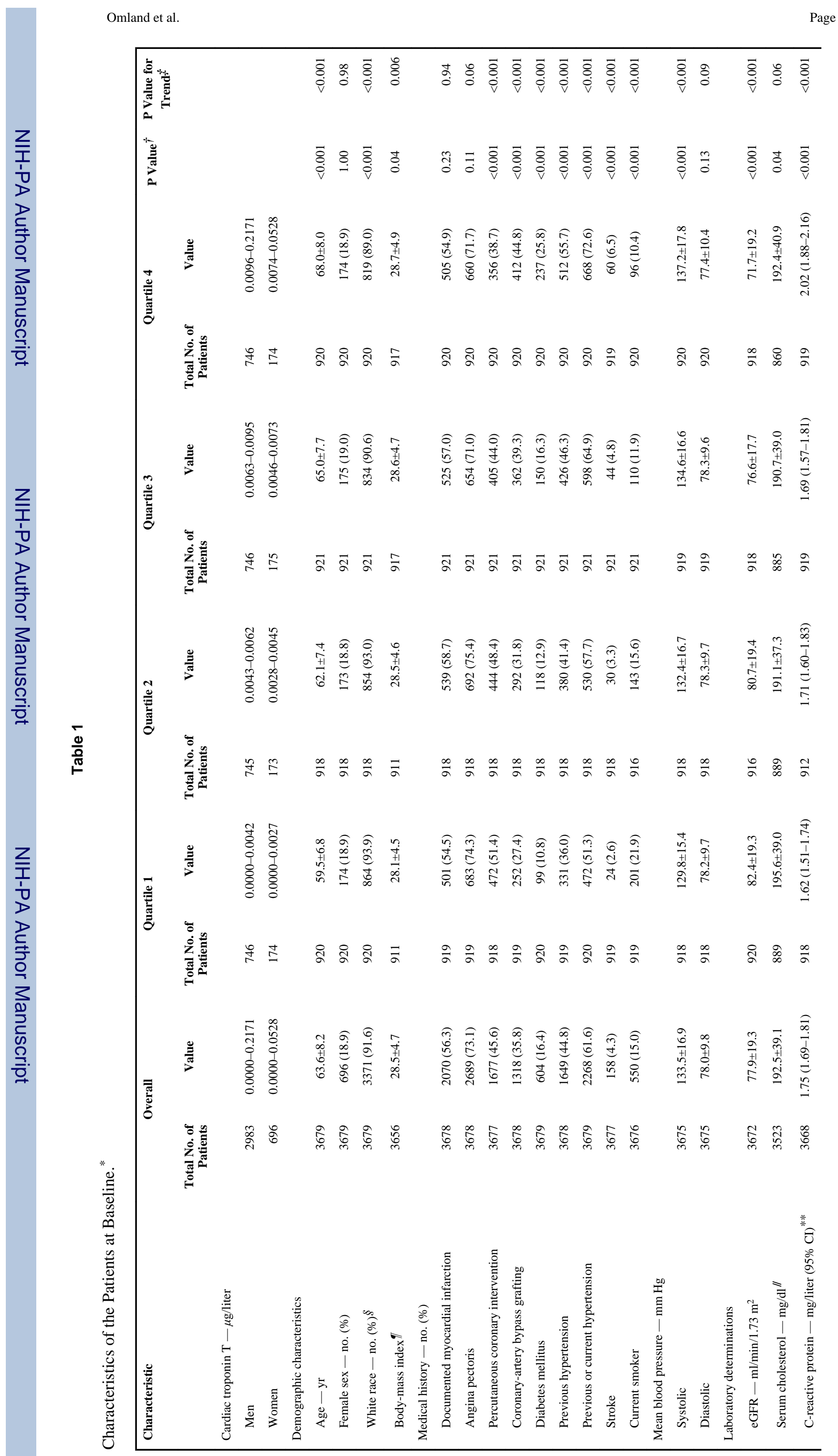




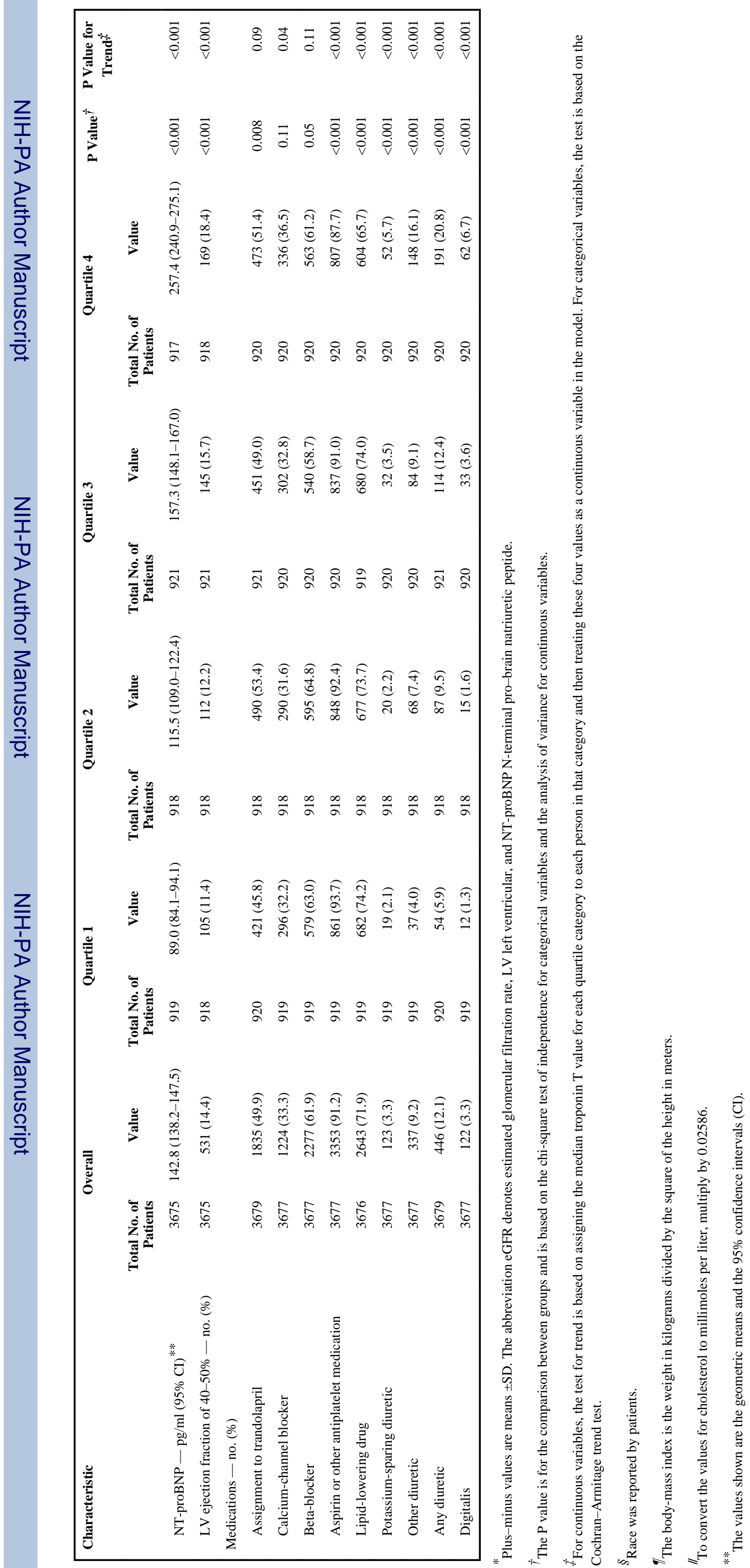




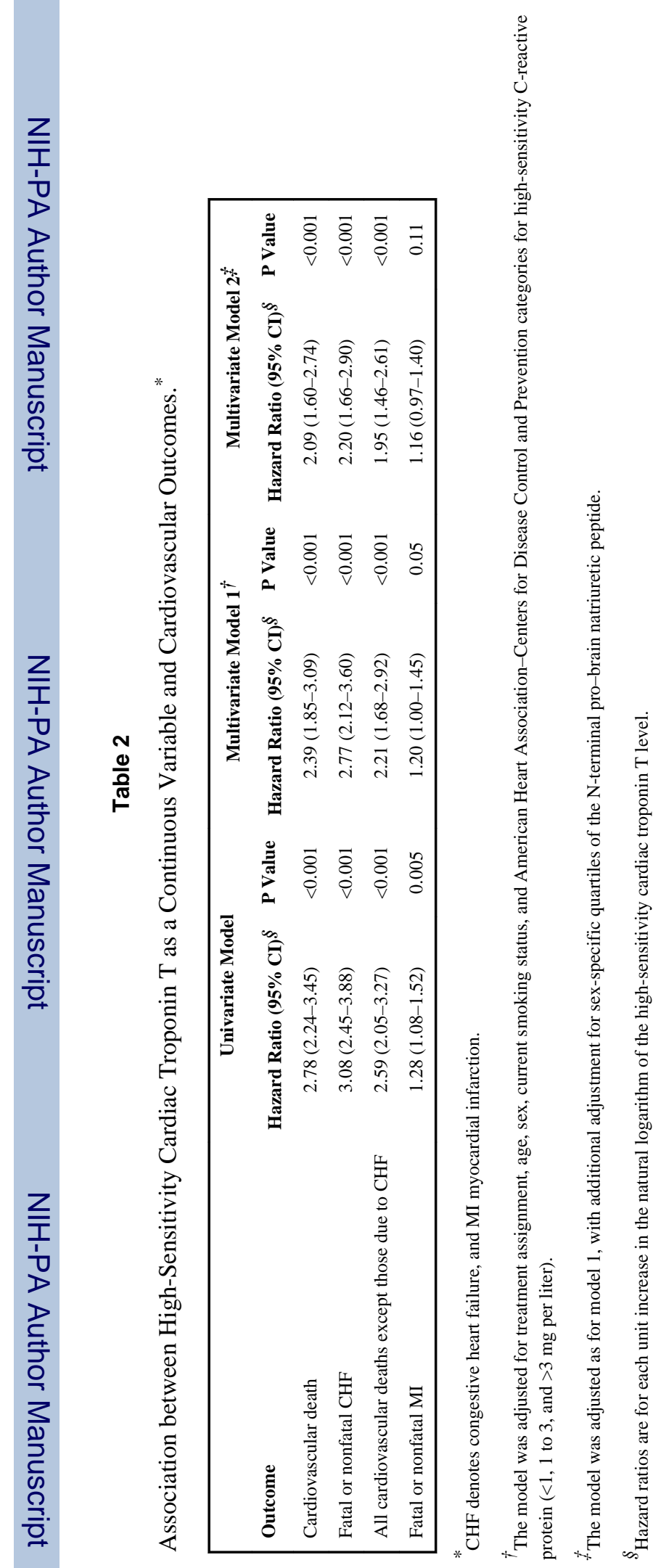

N Engl J Med. Author manuscript; available in PMC 2010 December 6. 